\title{
Passage of 10-keV Electrons through a Bundle of Dielectric Channels
}

\author{
K. A. Vokhmyanina ${ }^{a *}$, L. V. Myshelovka ${ }^{a}$, D. A. Kolesnikov ${ }^{a}$, V. S. Sotnikova ${ }^{a, b}$, \\ A. A. Kaplii ${ }^{a}$, A. S. Kubankin ${ }^{a, c}$, P. N. Zhukova ${ }^{d}$, and V. Yu. Ionidi ${ }^{e}$ \\ ${ }^{a}$ Belgorod State University, Belgorod, 308015 Russia \\ ${ }^{b}$ Belgorod State Technological University, Belgorod, 308012 Russia \\ ${ }^{c}$ Lebedev Physical Institute, Russian Academy of Sciences, Moscow, 119991 Russia \\ ${ }^{d}$ Belgorod Law Institute of the Ministry of Internal Affairs of the Russian Federation, Belgorod, 308024 Russia \\ ${ }^{e}$ Skobeltsyn Institute of Nuclear Physics, Moscow State University, Moscow, 119991 Russia \\ *e-mail:vokhmyanina@bsu.edu.ru \\ Received July 21, 2020; revised September 24, 2020; accepted September 24, 2020
}

\begin{abstract}
Passage of a 10-keV electron beam through a bundle of hollow polysulfone fiber with an inner channel diameter of $160 \pm 60 \mu \mathrm{m}$ was studied. The dependence of the electron beam that passed through the channels on the channel tilt angle with respect to the incident beam axis was measured. The fraction of electrons with an energy loss of less than $10 \%$ after passing the channels was also estimated.
\end{abstract}

Keywords: electron beam, dielectric channels, dielectric surface, characteristic radiation.

$10.1134 / \mathrm{S} 1063785021010132$

The possibility of controlling a beam of nonrelativistic charged particles using dielectric channels was first demonstrated in [1]. A Ne ${ }^{7+}$ ion beam with an energy of $3 \mathrm{keV}$ changed direction following the tilt of dielectric nanocapillaries formed in a polyethylene terephthalate (PET) film. The main part of the incident beam passed through channels without losing its initial energy and charge state even in the case of substantial channel tilt angles. This effect has been explained as being due to some of the beam ions hitting the channel walls charging them to a certain value [1]. The thus-formed charge distribution prevents further charging of the walls and provides contactless movement of the remaining part of ions along the channel axes. The surface charge distribution is in a self-consistent mode when the leaking a part of the charge is instantaneously compensated by ions of the incident beam. This model was the basis of the interpretation and explanation of subsequent experimental studies [2-4].

Similar experiments with electron beams demonstrated the existence of the guiding effect; however, the discovered specific features of the studied process [57] complicate the search of the possibility of its practical application. At present, there does not exist a consistent model explaining the observed results $[8,9]$ for electron beams.
This study presents the experimental results on passage of $10-\mathrm{keV}$ electrons through dielectric channels representing hollow polysulfone fibers assembled in a bundle. The bundle of channels was tilted with respect to the incident beam axis, and the beam current was measured at the output; also, the fraction of electrons that lost less than $10 \%$ of the initial energy was estimated. This information is required to study the possibility of using channels from the dielectric material under study as a beam optics element.

The general scheme of the experiment is shown in Fig. 1.

Electrons 4 generated by electron gun 1 traverse system of electromagnetic lenses 2 and collimator 3 with a diameter of $1 \mathrm{~mm}$ and reach the input of sample 5 fixed in the goniometer. Samples with a length of 10 and $4.75 \mathrm{~mm}$ were studied. Each sample (Fig. 1b) represents a bundle consisting of 30 polysulfone fibers; the inner diameter of each channel is $160 \pm 60 \mu \mathrm{m}$, and the wall thickness is $60 \mu \mathrm{m}$. Fibers are glued, and the whole bundle is covered by a heat-shrinking film. The input edge of the sample was covered by a thin layer of silver (up to $100 \mathrm{~nm}$ ) for preventing charging of channel inputs. The transparency of the final array was $\sim 20 \%$, the maximum geometric transmission angles of $\pm 1.26^{\circ}$ and $\pm 2.65^{\circ}$ were calculated for a maximum 


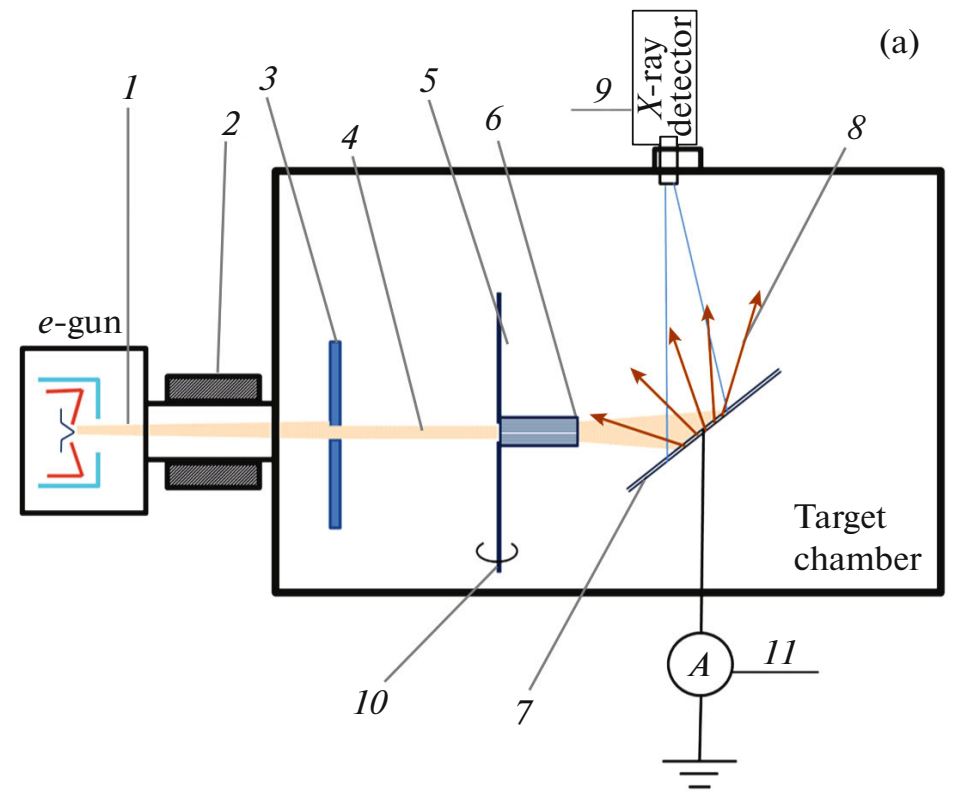

(b)

Fig. 1. (a) Scheme of experiment: (1) electron gun, (2) system of electromagnetic lenses, (3) collimator with a diameter of $1 \mathrm{~mm}$, (4) accelerated electron beam, (5) sample with a grounded mask, (6) electron beam traversed through the sample, (7) copper plate, $(8)$ radiation generated by electrons hitting the copper plate, $(9) \mathrm{X}$-ray detector, (10) sample tilt axis with respect to the incident beam axis, and (11) ammeter; (b) schematic diagram of the sample and photo of the output edge (without a metal deposited layer).
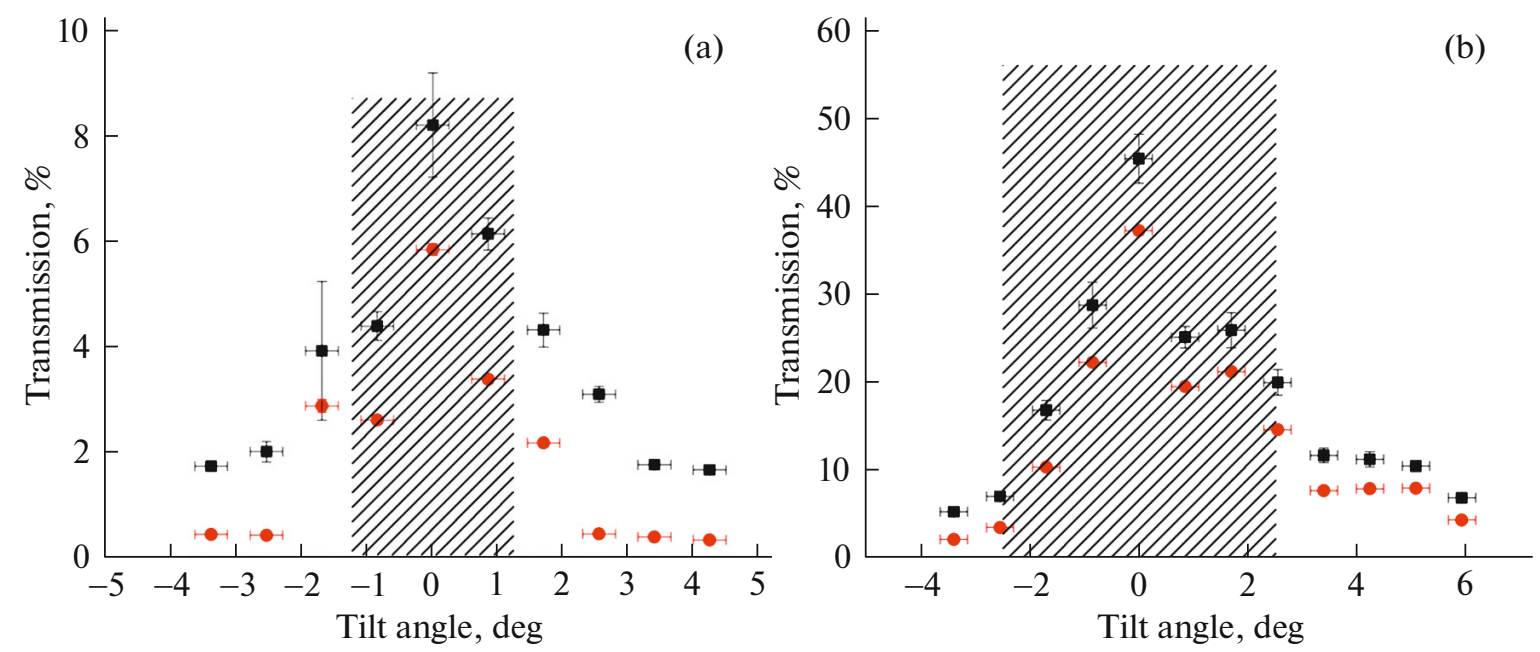

Fig. 2. (Squares) fractions of the beam passed through the channel and (circles) fractions of electrons with energy loss not exceeding $1 \mathrm{keV}$ as functions of channel tilt angles with respect to the incident beam axis. The channel length is (a) 10 and (b) $4.75 \mathrm{~mm}$.

channel diameter of $220 \mu \mathrm{m}$ and for sample lengths of 10 and $4.75 \mathrm{~mm}$, respectively.

The goniometer provides sample tilting with respect to the axis of the incident beam around axis 10 . Electrons, after passing channels, hit copper plate 7 , generating electromagnetic radiation 8 measured by X-ray detector (XR-1-SDD) 9 with a beryllium window with a thickness of $12.5 \mu \mathrm{m}$ and an effective area of $17 \mathrm{~mm}^{2}$. Spectra were recorded for $20 \mathrm{~min}$.
The beam current of transmitted electrons was measured simultaneously using nanoammeter (Keithley 6482) 11. The primary beam had a beam current of $100 \mathrm{nA}$, a beam diameter at the input of the array of $2 \mathrm{~mm}$, and a beam divergence of less than $\pm 0.3^{\circ}$. The pressure in the vacuum chamber was less than $10^{-6}$ Torr.

Figure 2 shows the measured beam current for electrons that propagated through dielectric bundles of channels as a function of sample tilt angle with 


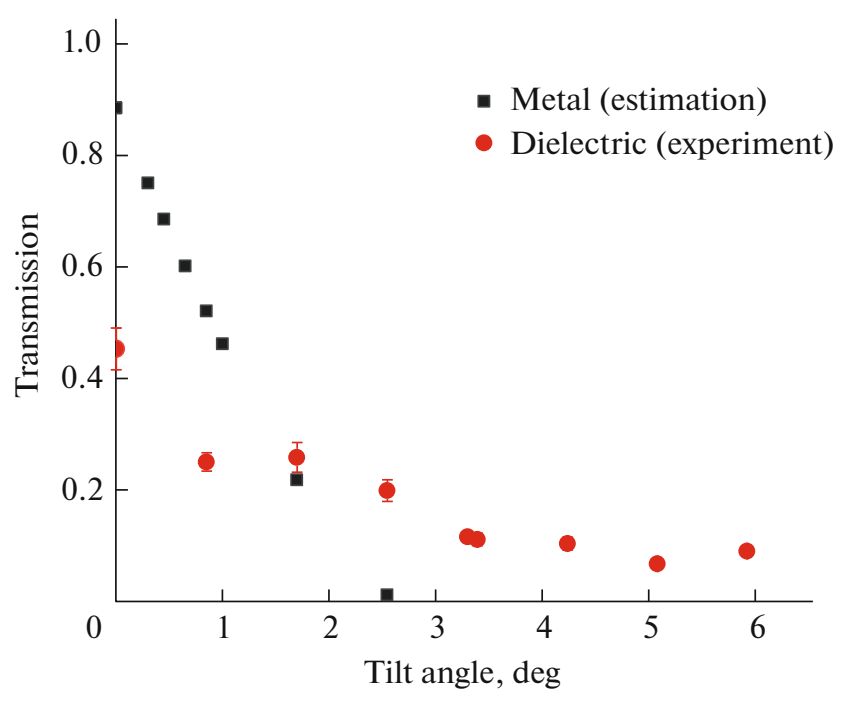

Fig. 3. Comparison of the fraction of incident electrons that passed through the dielectric (experiment, circles) and the metallic (calculations, squares) channels with the following geometric parameters: inner diameter $220 \mu \mathrm{m}$, length $4.75 \mathrm{~mm}$.

respect to the incident beam axis. It also shows the fraction of electrons of the incident beam with an energy loss of no more than $1 \mathrm{keV}$. This quantity was estimated using the method described in detail in [10]. The essence of the method lies in the comparison of the spectra of incident and passed through the channels electromagnetic radiation generated in interaction of electrons with the copper plate. The part of the spectrum connected with the copper characteristic $K_{\alpha}$ line (with a photon energy of $8.048 \mathrm{keV}$ ) is examined; for this line to appear, the electron energy should be at least $8.993 \mathrm{keV}$ ( $K_{\text {edge }}$ for copper). The method has an evaluative character, since it is assumed that the number of events of the spectrum linearly depends on the number of electrons hitting the copper plate.

The dashed rectangle is the region of geometric transmission of channels. The fraction of electrons is calculated with respect to the current incident on the channel with account of a bundle transparency of $20 \%$.

It can be seen from Fig. 2 that the reduction of the channel length by approximately a factor of 2 provided that there is fivefold growth of the fraction of passing current. In both cases, the reduction of transmission with increasing channel tilt angle has a similar character: the intensity of the passed beam reduces gradually at angles exceeding the geometric transmission angle. The comparison of the angular dependence for the considered dielectric channels with transmission of a metallic grounded channel (channel walls are not charged) for the same geometric parameters yields that, in the case of the metallic channel, the transmis- sion drops faster than in the case of the dielectric channel (Fig. 3).

The data for the metallic channel were calculated using formula (65) from [11] taking into account used beam parameters. This result unambiguously indicates the influence of the dielectric wall, namely, the surface charge distribution, on the electron motion, i.e., a small fraction of electrons is not captured by the wall, as in the case of the metallic channel, but continues to move along the channel axis without a noticeable energy loss even in the case of substantial tilt angles. The asymmetry of the dependences obtained in Fig. $2 \mathrm{~b}$ can be explained by nonsymmetric charging of inner walls of the channel, since the channel was tilted in one direction from the maximum tilt angle in the experiment. More precise description of the observed dependence, however, requires a separate study.

The experimental results demonstrated the effect of the surface charge on the channel walls on the propagating electron beam, and, therefore, the possibility of controlling electrons with an energy of $10 \mathrm{keV}$ using such structures. The dependence of the fraction of transmitted beam on the channel length in the bundle was also explicitly demonstrated. With a decrease in the channel length by about a factor of 2 , the fraction of transmitted beam increased by approximately a factor of 5. The fraction of electrons that propagated without a contact for the tilt angle equal to the geometric opening angle reduced on average by a factor of 2.5 as compared to that for the zero tilt angle for both channels. Thus, it can be stated that a $10-\mathrm{keV}$ electron beam can be guided using channels from polysulfone fiber in a small angular range.

\section{FUNDING}

The work was financially supported by the context part of a state order for creation and development of laboratories, project no. FZWG-2020-0032 (2019-1569).

\section{CONCLIFT OF INTEREST}

The authors declare that they have no conflict of interest.

\section{REFERENCES}

1. N. Stolterfoht, J. H. Bremer, V. Hoffmann, D. Fink, R. Hellhammer, A. Petrov, and B. Sulik, Phys. Rev. Lett. 88, 133201 (2002).

2. T. Nebiki, T. Yamamoto, T. Narusawa, M. B. H. Breese, E. J. Teo, and F. Watt, J. Vac. Sci. Technol. A 21, 1671 (2003).

3. V. Mäckel, W. Meissl, T. Ikeda, M. Clever, E. Meissl, T. Kobayashi, T. M. Kojima, N. Imamoto, K. Ogiwara, and Y. Yamazaki, Rev. Sci. Instrum. 85, 014302 (2014). 
4. P. Skog, H. Q. Zhang, and R. Schuch, Phys. Rev. Lett. 101, 223202 (2008).

5. S. Das, B. S. Dassanayake, M. Winkworth, J. L. Baran, N. Stolterfoht, and J. A. Tanis, Phys. Rev. A 76, 042716 (2007).

6. Y. Xue, D. Yu, J. Liu, M. Zhang, B. Yang, Y. Zhang, and X. Cai, Appl. Phys. Lett. 107, 254102 (2015).

7. S. J. Wickramarachchi, T. Ikeda, D. Keerthisinghe, B. S. Dassanayake, and J. A. Tanis, Nucl. Instrum. Methods Phys. Res., Sect. B 317, 101 (2013).

8. K. Schiessl, K. Tokési, B. Solleder, C. Lemell, and J. Burgdörfer, Phys. Rev. Lett. 102, 163201 (2009).
9. A. R. Milosavljević, J. Jureta, G. Vikor, Z. D. Pešić, D. Šević, M. Mátéfi-Tempfli, S. Mátéfi-Tempfli, and B. P. Marinković, Europhys. Lett. 86, 23001 (2009).

10. K. A. Vokhmyanina, A. S. Kubankin, L. V. Myshelovka, H. Zhang, A. A. Kaplii, V. S. Sotnikova, and M. A. Zhukova, J. Instrum. 15, C04003 (2020).

11. V. N. Stolterfoht and Y. Yamazaki, Phys. Rep. 629, 1 (2016).

Translated by E. Baldina 